\section{Wip1 regulates hematopoietic stem cell development in the mouse embryo}

The first adult-repopulating hematopoietic stem cells (HSC) are found in the aorta-gonad-mesonephros (AGM) region at embryonic day (E) $10.5,{ }^{1,2}$ as well as in other sites, such as the embryonic head, ${ }^{3,4}$ yolk sac (YS) and placenta. $^{5-7}$ Accumulating evidence from ex vivo models suggests that HSC emerge from endothelial cells (EC) of the aorta by forming sprouting clusters, ${ }^{8-10}$ through the preHSC I (VE-Cadherin ${ }^{+} \mathrm{CD} 41^{\text {low }} \mathrm{CD}^{-} 5^{-}$) and pre-HSC II (VECadherin ${ }^{+} \mathrm{CD} 45^{+}$) stages of maturation, and are regulated by various transcription factors and signaling pathways. Less is known about the exact mechanism of maturation of pre-HSC in vivo. Wild-type p53-induced phosphatase (Wip1) is defined as a critical regulator involved in HSC function and lymphoid/neutrophil development. ${ }^{11,12}$ However, the role of Wip1 in regulating HSC function and pre-HSC maturation in the embryo is still unknown.

Our data have shown that Wip1 is expressed in EC and pre-HSC in the AGM region and fetal liver (FL)/bone marrow HSC, ${ }^{13}$ suggesting that Wip1 may be involved in hematopoiesis (Online Supplementary Figure S1A). To test whether Wip1 plays a role in hematopoietic development of the embryo, Wip1 homozygous deficient embryos $\left(\right.$ Wip $^{-1}$, KO) were used in this study. The total cell number was significantly decreased in E12.5 $\mathrm{Wip}^{-1-} \mathrm{FL}$ compared with wild-type (Wip ${ }^{+/+}$, WT) $\left(2.8 \pm 0.4 \times 10^{6}\right.$ vs. $\left.4.1 \pm 0.5 \times 10^{6}\right)$ and $\operatorname{E13.5}\left(5.9 \pm 0.5 \times 10^{6}\right.$ vs. $\left.9.1 \pm 0.7 \times 10^{6}\right)$ (Figure 1A, Online Supplementary Figure 1B). The percentages of $\mathrm{CD} 45^{+}$and $\mathrm{CD} 34^{+} \mathrm{c}-\mathrm{Kit}^{+}$cells were indistinguishable, but the absolute cell numbers of both populations were obviously reduced, respectively (Figure 1B-E, Online Supplementary Figure $S 1 C, D)$. To test the hematopoietic progenitor cell (HPC) function, colony-forming unit-culture (CFU-C) assays were performed, and revealed smaller size of burst-forming unit-erythroid (BFU-E), CFUgranulocyte macrophage (CFU-GM) and CFU-granulocyte erythrocyte monocyte megakaryocyte (CFU-Mix) colonies in the E12.5 Wip $1^{-1-}$ FL compared with E12.5 WT FL (Online Supplementary Figure S1E). The number of CFU-C per E12.5 Wip $1^{-1-}$ FL was reduced by more than $70 \%\left(3.4 \pm 0.3 \times 10^{3} \mathrm{E} 12.5\right.$ vs. $\left.14.2 \pm 1.4 \times 10^{3}\right)$, with dramatic reductions in BFU-E, CFU-GM and CFU-Mix numbers. The same trend in reduced CFU-C numbers was observed from E13.5 to E14.5 in Wip $1^{-1}$ FL. Interestingly, the CFU-C numbers from the same input number of FL cells $\left(2 \times 10^{4}\right.$ cells) were decreased considerably (Figure 1F, G, Online Supplementary Figure S1F), demonstrating the influence of Wip1 deletion on the potential of HPC in the FL.

Subsequently, flow analysis and transplantation were used to investigate Wip1 function in FL HSC development. Firstly, there was a significant reduction in the percentage of HSC of E12.5 Wip 1 FL compared to WT FL $(0.015 \pm 0.003 \%$ vs. $0.025 \pm 0.004 \%)$ as revealed by a cocktail of markers ( $\mathrm{Lin}^{-} \mathrm{CD} 48^{-} \mathrm{Mac} 1^{\text {low }} \mathrm{Sca}-1^{+} \mathrm{CD} 150^{+}$, SLAM HSC). Moreover, the absolute number of SLAM HSC was reduced by more than $30 \%(419 \pm 80$ vs. $607 \pm 116 /$ embryo equivalent), similar to the decrease in the percentage and absolute number of $\mathrm{Lin}^{-} \mathrm{Mac} 1^{\text {low }} \mathrm{Sca}-1^{+}$cells (including more immature HPC) (Figure 1H, J, Online Supplementary Figure $S 2 A, B)$. Subsequently, assays following in vivo transplantation by injection of 0.05 embryo equivalent/recipient revealed that six out of 19 recipients given E12.5 Wip1 ${ }^{-/} \mathrm{FL}$ cells were repopulated, with $12.7 \pm 4.6 \%$ chimerism, whereas 15 out of 17 recipients of WT cells achieved chimerism of $65.0 \pm 7.8 \%$ (Figure $1 \mathrm{~K}$
Online Supplementary Table S1). Multilineage assays showed the reduction of B-cell lineage output (B220 \%) in the peripheral blood from Wip $1^{-/}$FL derived-recipients. (Figure 1M, Online Supplementary Figure S2C-G). Secondary transplantation data showed that HSC selfrenewing ability was decreased in the Wip $1^{-1}$ group (Online Supplementary Figure S2H). Although all the recipients were repopulated, the average chimerism was decreased in the E14.5 Wip $1^{-1} \mathrm{FL}$, with reductions in Band T-cell lineage output and an increase of myeloid cells (Figure 1L, N). Taken together, our findings indicate the involvement of Wip1 in HPC development and HSC activity of embryonic FL with a decrease in lymphoid lineage differentiation.

E11.5 embryos with Wip1 deficiency are smaller than their WT littermates (Online Supplementary Figure S3A). The total cell number was decreased from E10.5 to E12.5 AGM (Online Supplementary Figure S3B). Flow analysis data showed that the percentages of CD45 were comparable in the Wip1 ${ }^{-1}$ AGM and YS and the corresponding WT tissues; however, the percentage of CD41 $1^{\mathrm{low}} \mathrm{CD} 45^{-}$ cells was decreased significantly in the E11.5 AGM region, but not in the YS (Online Supplementary Figure $S 3 C-F$ ), indicating a reduction of HPC. CFU-C assays confirmed that Wip1 ablation leads to a dramatic decrease in HPC function $(61 \pm 21$ vs. $326 \pm 49$ CFU$\mathrm{C} / \mathrm{AGM}$ ) at E10.5, including reduced numbers of CFUMix and CFU-GM. Similar decreases were found in the E11.5 and E12.5 Wip1 ${ }^{-1}$ AGM $(85 \pm 13$ and $30 \pm 8$ CFUC/AGM, respectively) compared with WT AGM $(249 \pm 46$ and 208 \pm 39 CFU-C/AGM, respectively) (Figure 2A). High proliferative potential colony-forming cells presenting immature HPC were clearly reduced in the Wip $1^{-1}$ AGM (Online Supplementary Figure S3G). Meanwhile, similar trends in reduction were found in E10.5-E12.5 $\mathrm{Wip}^{-1 /} \mathrm{YS}$ (Figure $2 \mathrm{~B}$ ). These results suggest a positive regulatory role of Wip1 in HPC function in the AGM region and YS.

Thereafter, in vivo transplantation assays were performed to test the influence of Wip1 on HSC functions. Four of $12(33 \%)$ recipients of E11.5 WT AGM cells showed long-term, high-level multilineage repopulation, but none of nine recipients of Wip ${ }^{-1}$ AGM cells were repopulated. Unexpectedly, two of seven recipients of E12.5 $\mathrm{Wip}^{-1}$ AGM cells were repopulated, with a lower repopulation ratio compared with the WT group $(6 / 6$ recipients were repopulated). The $\mathrm{B}$-cell lineage output was diminished in Wip1 ${ }^{\%}$ AGM-derived recipients (Figure 2C, D, Online Supplementary Table S1), consistent with Wip ${ }^{-1}$ FL and bone marrow HSC. In E11.5 YS, the ability to engraft was comparable (3/9 with chimerism of $9.1 \pm 4.1 \%$ vs. $2 / 6$ with chimerism of $15.4 \pm 10.5 \%$ ); however, the engraftment ability was decreased significantly in E12.5 $\mathrm{Wip}^{-1-}$ YS with an increase of myeloid output (8/11 vs. 9/9) (Figure 2E, F). These data, together with the HPC results, indicate that Wip1 affects hematopoietic stem and progenitor cell function mainly in the E11.5 AGM region.

HSC are reported to emerge autonomously at E10.5 (>35 somite pairs) in the AGM region but are inefficient in direct transplantation because of their low activity. ${ }^{1}$ Explant culture is an efficient tool for HSC expansion/pre-HSC maturation. Explant culture assays revealed a decrease in HSC activity in the recipients receiving E10.5 Wip1\% AGM (4/9) compared with WT AGM (11/12), with a greater myeloid output at the cost of $\mathrm{T}$ lymphoid output (Figure 2G, H), indicating that Wip1 ablation may affect HSC expansion and/or preHSC maturation.

We also investigated whether Wip1 influences pre- 
A

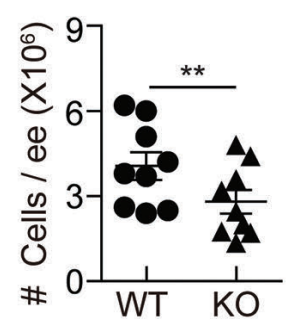

$\mathrm{F}$

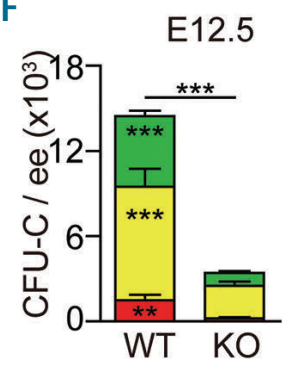

H
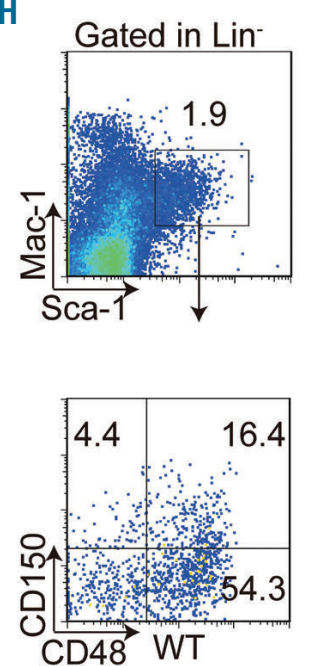

B

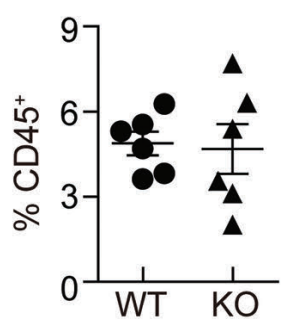

C

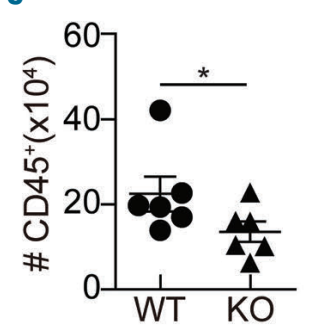

D

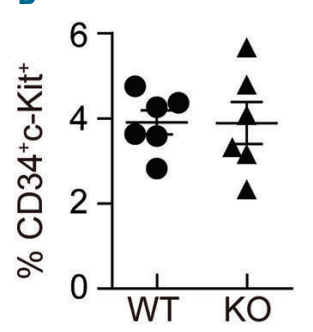

E

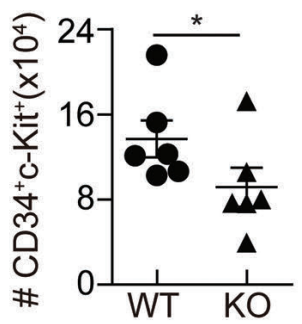

G

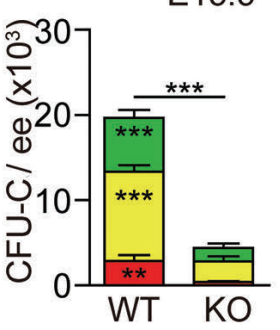

E14.5
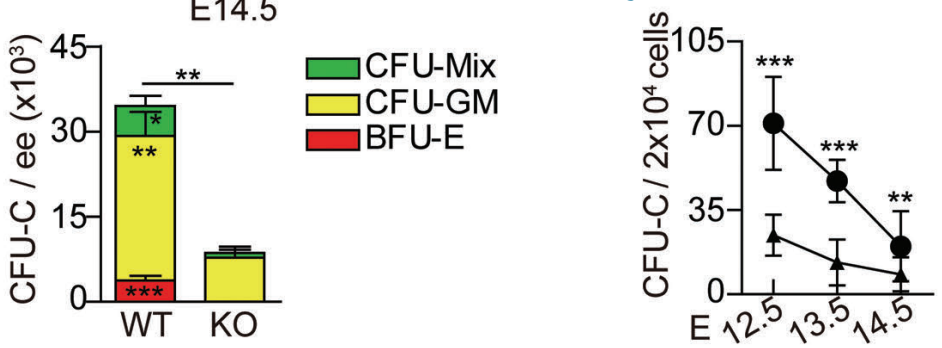

I

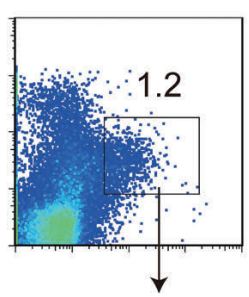

K
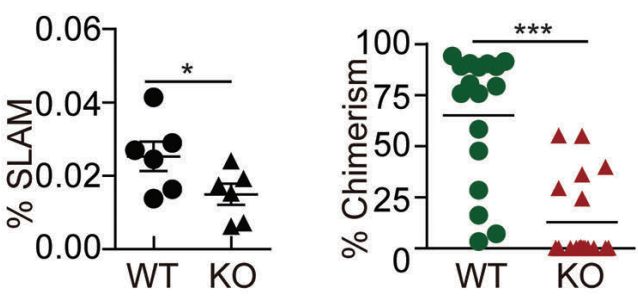

M

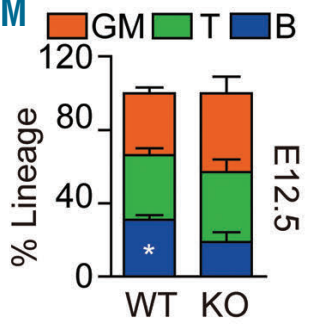

L
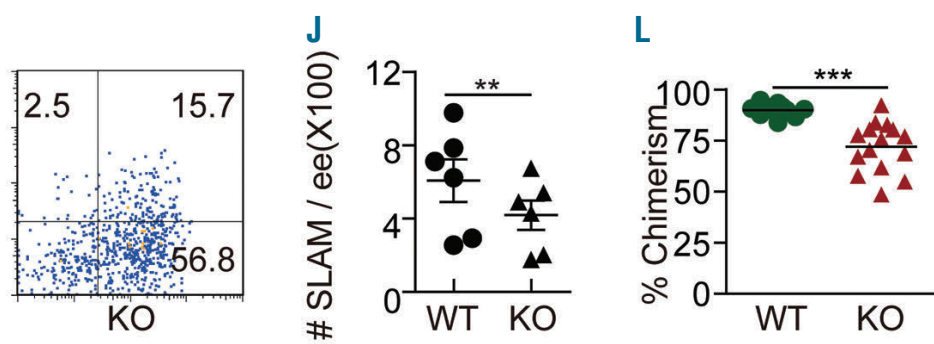

N

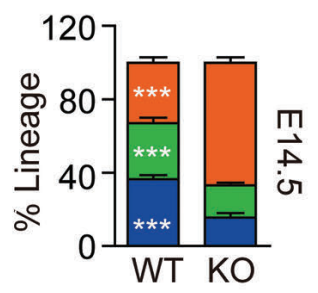

Figure 1. Wip1 knockout resulted in the reduction of hematopoietic progenitor cell number and hematopoietic stem cell activity in embryonic day $12.5-14.5$ fetal liver. (A) The reduction of cell number on embryonic day (E) 12.5 Wip $1 \%$ fetal liver (FL) $(n=9, * * P=0.0028)$. (B-E) Percentages (B, D) and absolute numbers $(\mathrm{C}, \mathrm{E})$ of $\mathrm{CD}_{4} 5^{+}$and $\mathrm{CD} 34^{+} \mathrm{C}-\mathrm{Kit}^{+}$cells on E12.5 $\left(\mathrm{n}=6,{ }^{*} \mathrm{P}<0.05\right)$. $(\mathrm{F}, \mathrm{G})$ Colony-forming unit - culture assay showed the number of colonies per $\mathrm{FL}(\mathrm{F})$ and per $2 \times 10^{4}$ cells $(\mathrm{G})$ from E12.5 to E14.5. Colony types are indicated by colored bars. E12.5, E13.5, and E14.5: $n=3,3$, and 2, respectively; $* P<0.05$, $* * P<0.01$, $* * * P<0.001$. (H) Representative flow cytometric analysis of phenotypic hematopoietic stem cells (HSC) (Lin-Mac- ${ }^{1 \text { ow }} \mathrm{Sca}-1^{+} \mathrm{CD} 150^{+} \mathrm{CD} 48^{-}, \mathrm{SLAM}$ HSC). (I, J) Percentage (I) and number $(J)$ of SLAM HSC in the E12.5 wild-type (WT) and Wip $1 \% \mathrm{FL}(\mathrm{n}=6, * P=0.0189$ and $* * P=0.0017)$. (K, L) Reconstituting potential of E12.5 and E14.5 WT and Wip1\% FL. Chimerism (\%CD45.2 ) in peripheral blood of recipients at 16 weeks after transplantation. Each symbol represents one recipient. Green circle $=W T$, red triangle $=$ Wip $1 \%$. The lines represent the average chimerism $(n=3, * * * P<0.001$. (M, N) Multilineage output of peripheral blood of representative repopulated recipients. $(n=3, * P=0.02$ and $* * * P<0.001)$. ee: embryo equivalent; WT: wild-type; KO: knockout; CFU-C: colony-forming unit culture; CFU-Mix: colony-forming unit - granulocyte, erythrocyte, monocyte, megakaryocyte; CFU-GM: colony-forming unit - granulocyte-macrophage; BFU-E: burstforming unit - erythroid; GM: Gr-1 $1^{+} / \mathrm{Mac}_{-1} 1^{+}$cells; T: $\mathrm{CD} 3^{+}$cells; B: B220 ${ }^{+}$cells.

HSC maturation. $\mathrm{CD} 31^{+} \mathrm{CD} 41^{\text {low }} \mathrm{CD} 45^{-}$(pre-HSC I) and $\mathrm{CD} 31^{+} \mathrm{CD} 45^{+}$(pre-HSC II) cells were characterized by flow analysis. The percentages of pre-HSC I were comparable in the E11.5 Wip1 ${ }^{-1}$ AGM and WT AGM $(0.116 \pm 0.030 \%$ vs. $0.079 \pm 0.009 \%)$ as were the absolute number of cells $(207 \pm 44$ vs. $176 \pm 22$, respectively) (Figure $3 \mathrm{~A}-\mathrm{C})$. However, Wip1 deletion resulted in reduced percentages of HSC II $(0.037 \pm 0.004 \%$ vs. $0.054 \pm 0.006 \%)$ and greater than $40 \%$ decreases of absolute numbers of pre-HSC II (68 \pm 9 vs. 121 \pm 15 , respectively) (Figure 3A, D, E). The trends were similar when CD201 was included (Online Supplementary Figure $S 4 A-D$ ). To test the potential of pre-HSC, pre-HSC I and pre-HSC II from E11.5 AGM were co-cultured with OP9-DL1 cells for 6 days prior to transplantation assays. The ratio of repopulated recipients was decreased slightly in the Wip $1^{-/}$pre-HSC I cultures $(2 / 6 ; 33 \%)$ compared with WT cultures $(3 / 4 ; 75 \%)$, with lower B lymphoid and higher myeloid lineage output. Meanwhile, 5/16 recipients were reconstituted by injecting $\mathrm{Wip}^{-/}$pre-HSC II cultures with lower chimerism $(14.3 \pm 5.6 \%)$ and higher myeloid lineage in comparison to WT recipients (6/12, with $27.6 \pm 8.7 \%$ chimerism) (Figure 3F-G, J-K). To further confirm the roles of Wip1 in pre-HSC maturation, a Wip1 inhibitor 
(CCT007093, CCT) was added. None of the recipients that received pre-HSC I cultures with inhibitor was repopulated, whereas six of seven recipients given dimethylsulfoxide (DMSO) instead of the inhibitor achieved chimerism $(33.3 \pm 9.0 \%)$. In the pre-HSC II cultures, the presence of the CCT inhibitor reduced the ability of engraftment of pre-HSC II-derived HSC cultures compared to the cultures with DMSO (5/10 [50\%] vs. 7/9 $[77.8 \%]$; chimerism $13.5 \pm 5.2 \%$ vs. $46.2 \pm 8.8 \%$, respec- tively) (Figure $3 \mathrm{H}, \mathrm{I})$. Additionally, the lymphoid lineage output of pre-HSC II cultures was changed in the presence of the Wip1 inhibitor (Figure 3L, M). These results, together with the direct transplantation findings, indicate that Wip1 educates the maturation of pre-HSC in the AGM region.

Hematopoietic stem and progenitor cells emerge from the EC of the aorta, by forming 'hematopoietic clusters, ${ }^{8,10,14}$ including pre-HSC, HSC and HPC. Here, Wip1
A
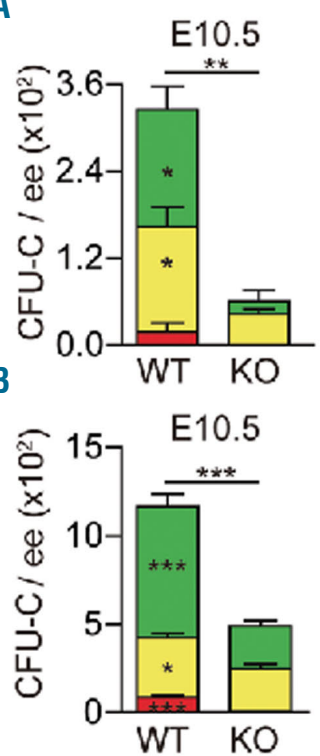

C

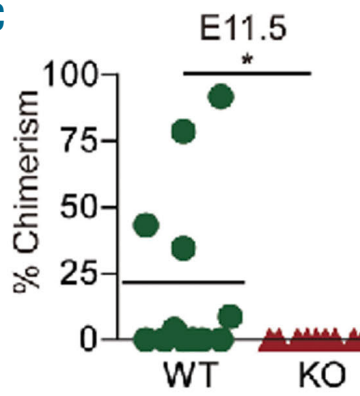

$E$
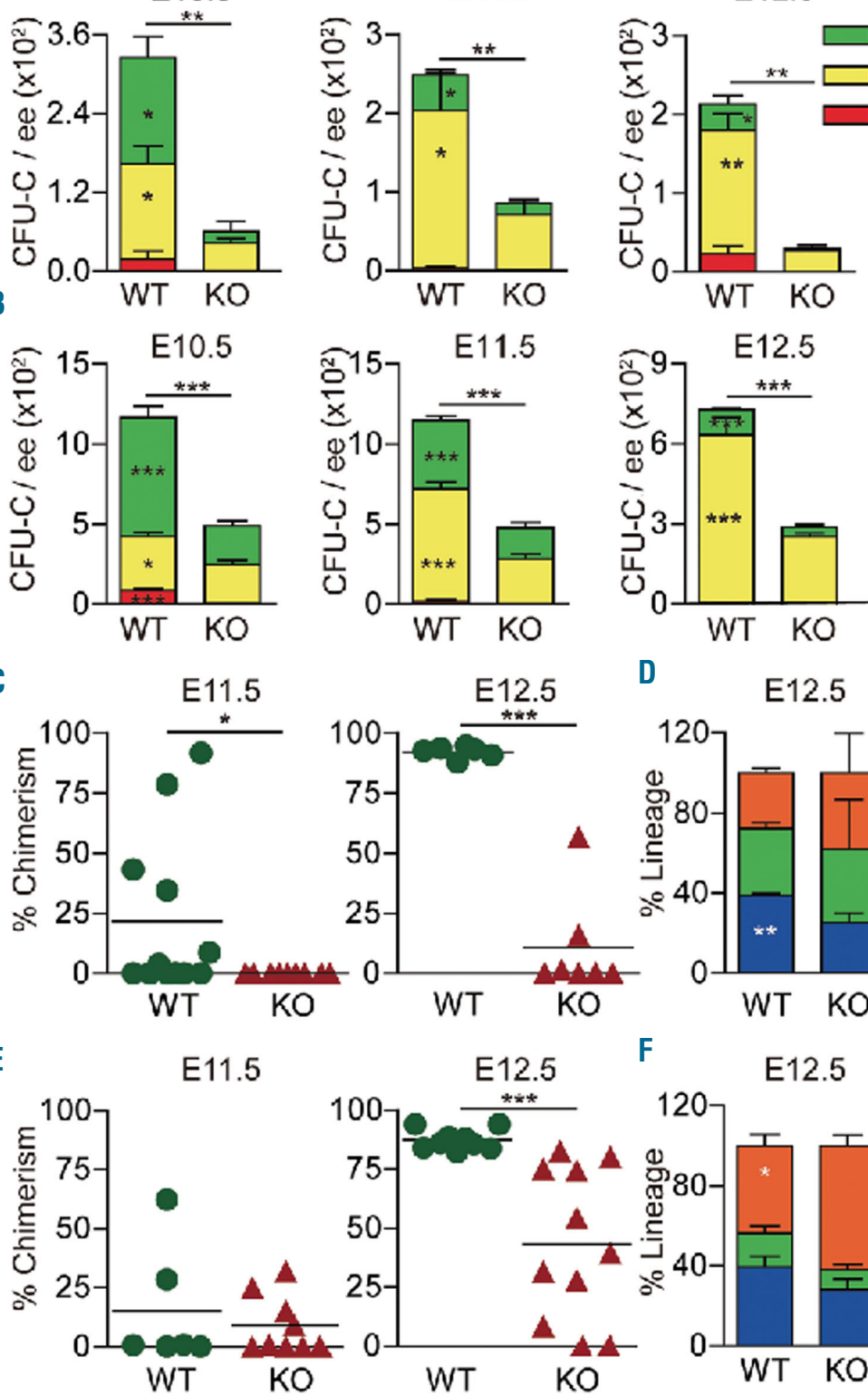

E12.5

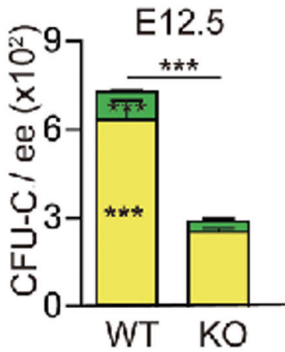

D
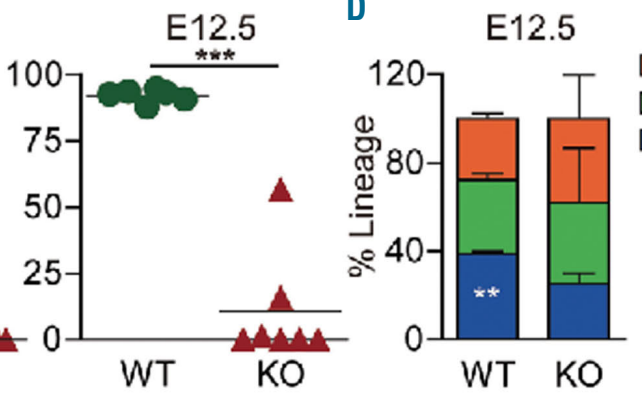

F
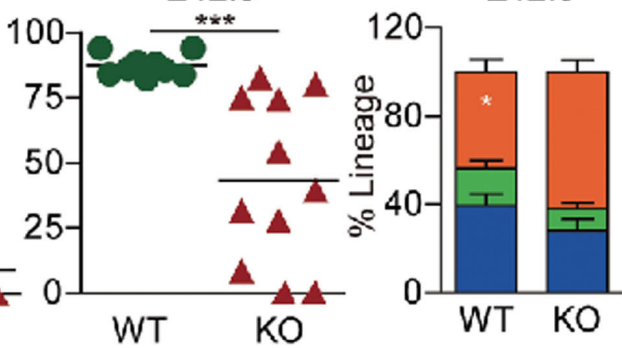

G

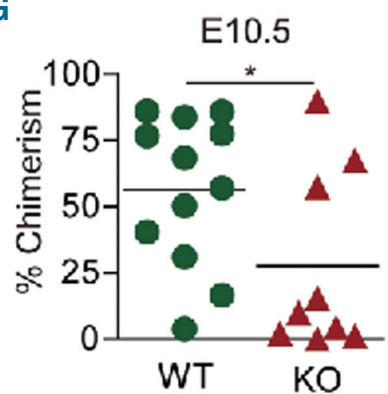

$\mathrm{H}$

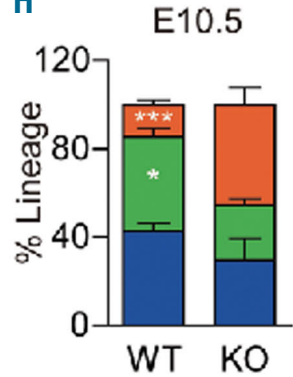

CFU-Mix

CFU-GM

AGM

$\mathrm{AGM}^{\mathrm{ex}}$

Figure 2. Hematopoietic stem and progenitor cell function was reduced in the embryonic day 10.5-12.5 Wip1\% aorta-gonadmesonephros region and yolk sac. $(A, B)$ Colony-forming unit culture assay showed the number of colonies in the aorta-gonadmesonephros (AGM) (A) and yolk sac (YS) (B) on embryonic day (E)10.5, E11.5 and E12.5 $(* P<0.05, \quad * * P<0.01$, $* * * P<0.001$ for wild-type [WT] vs. Wip 1 ) (C) Direct transplantation assay showed the repopulating ability of E11.5 and E12.5 WT and Wip $1 \%$ AGM. E11.5 $(n=4)$ and E12.5 $(\mathrm{n}=3): \quad * P=0.03$ and $* * * P<0.001$. (D) The lineage output of donor-derived granulocytemacrophage (GM), T and B cells in E12.5 WT and Wip1\% AGM at 16 weeks after transplantation $(n=3, \quad * * P=0.0075)$. (E) Repopulating ability of E11.5 and E12.5 WT and Wip1 YS by direct transplantation. $(n=3$, $* * * P=0.0004)$. (F) Donor-derived multilineage output in the peripheral blood of repopulated recipients of GM, T and B cells repopulated by E12.5 YS at 16 weeks after transplantation $(n=3$, *P=0.017). (G) Repopulating activity of E10.5 WT and Wip1 AGM after 3 days of explant culture in vitro $\left(\mathrm{AGM}^{\mathrm{ex}}\right)$. ${ }^{*} \mathrm{P}=0.0237$. (H) Donor-derived lineage output of GM, T and B cells repopulated by E10.5 WT and Wip1\% AGM explants at 16 weeks after transplantation. $\quad * P=0.0106$, $\star * * P<0.001$. Each symbol representes one recipient. Green circles $=$ WT, Red triangles $=$ Wip $1 \%$ The lines represent average chimerism. CFU-C: colony-forming unit culture; ee: embryo equivalent; WT: wild-type; KO: knockout; CFU-Mix: colony-forming unit granulocyte, erythrocyte, monocyte, megakaryocyte; CFU-GM: colony-forming unit - granulocytemacrophage; BFU-E: burst-forming unit - erythroid; GM: Gr$1^{+} / \mathrm{Mac}^{+} 1^{+}$cells; $\mathrm{T}: \mathrm{CD}^{+}$cells; $\mathrm{B}$ : B220 ${ }^{+}$cells. 
ablation decreased the percentage of $\mathrm{CD} 31^{+} \mathrm{c}-\mathrm{Kit}^{\mathrm{high}}$ hematopoietic clusters in the E11.5 AGM $(0.087 \pm 0.005 \%$ vs. $0.111 \pm 0.006 \%$ ). Consistently, a greater than $35 \%$ reduction in the number of $\mathrm{CD} 31^{+} \mathrm{c}-\mathrm{Kit}^{\text {high }}$ cells was observed in the E11.5 Wip1 ${ }^{-/}$AGM $(159 \pm 10$ vs. $249 \pm 14$
cells/AGM, respectively) (Figure $3 \mathrm{~N}, \mathrm{O}$ ). Moreover, the hematopoiesis-related genes P2-Runx1 (a proximal P2 promoter of Runx1) and Gata2 were reduced significantly after the deletion of Wip1 in the E11.5 AGM clusters but increased in the E11.5 YS (Online Supplementary Figure
A
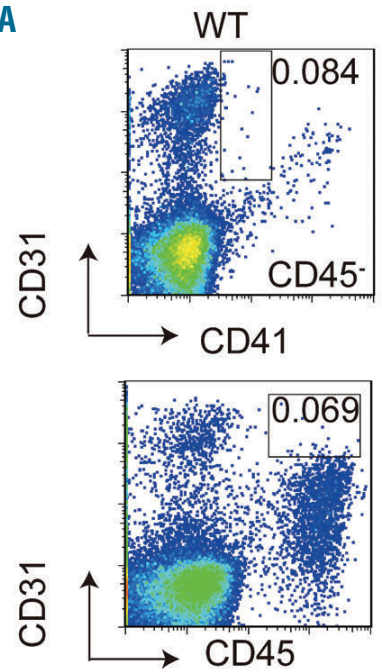

$\mathrm{F}$

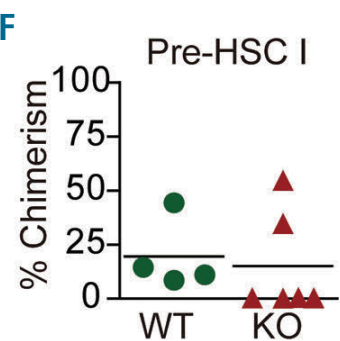

J

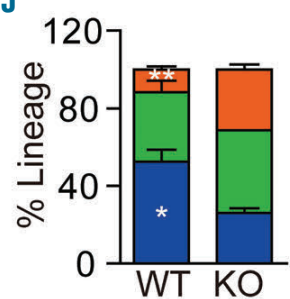

N

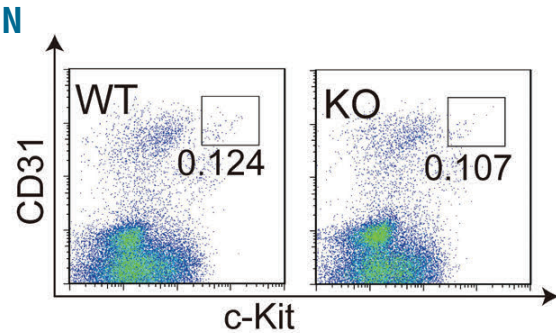

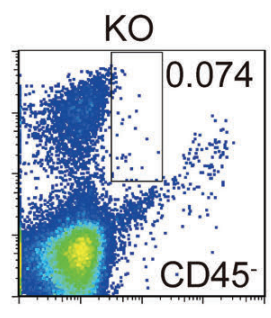

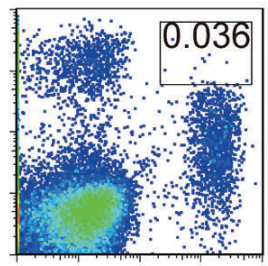

D
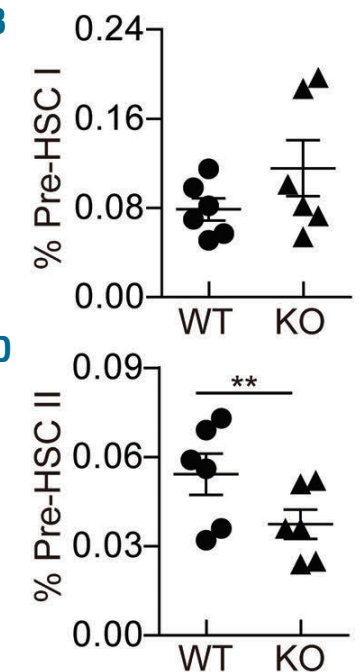

$\mathrm{H}$

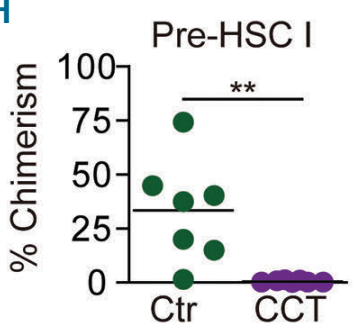

$\mathbf{L}$

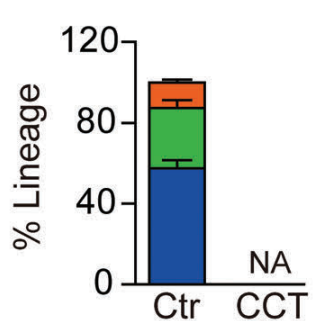

C

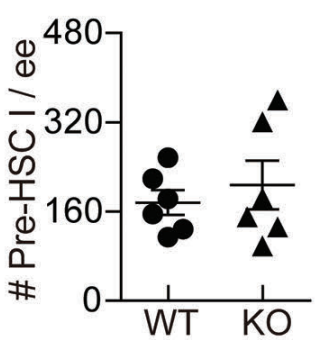

E
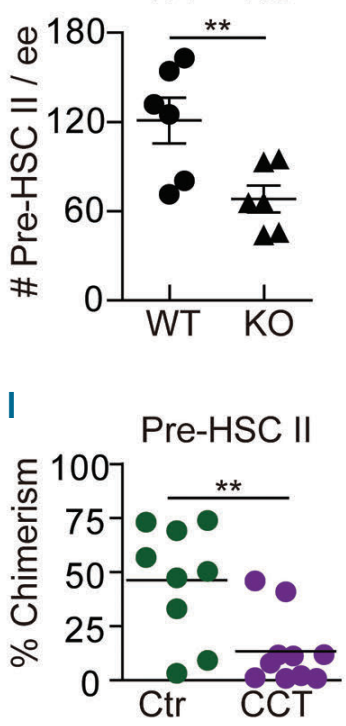

M

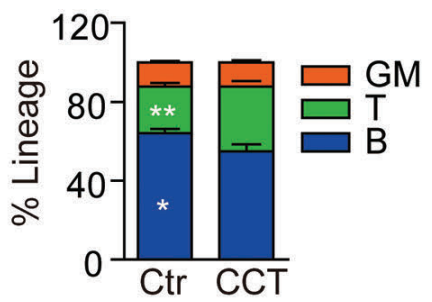

0

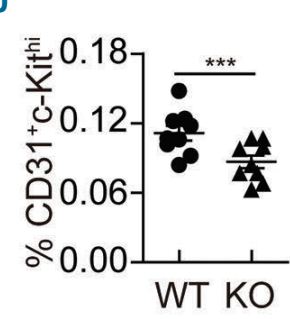

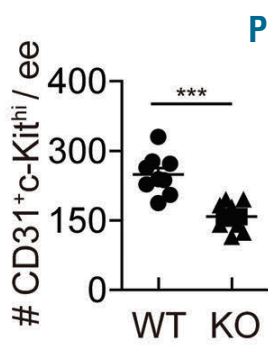

P

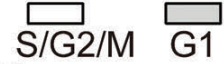

G0

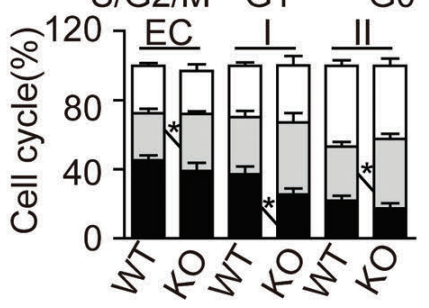

Figure 3. Pre-hematopoietic stem cell maturation was impeded by Wip1 ablation in the aorta-gonad-mesonephros region on embryonic day 11.5 . (A) Flow cytometric analysis showing the percentages of pre-hematopoietic stem cell (HSC) I (CD31 $1^{+}$CD $\left.41^{\text {low }} \mathrm{CD} 45^{-}\right)$and pre-HSC II (CD31 $\left.1^{+} \mathrm{CD} 45^{+}\right)$in wild-type (WT) and Wip1 aorta-gonad-mesonepros on embryonic day (E)11.5. (B-E) The percentages and absolute numbers of pre-HSC I (B and C, respectively) and pre-HSC II (D and $\mathrm{E}$, respectively), respectively. $\mathrm{n}=6, * * P<0.01$. (F, G) The repopulating potential of pre-HSC I (CD31 $\left.{ }^{+} \mathrm{CD} 45^{-}\right)$and pre-HSC II (CD31 $\left.{ }^{+} \mathrm{CD} 45^{+}\right)$from E11.5 WT and Wip1 AGM regions after co-culture with OP9-DL1 cells for 6 days. Chimerism was determined in the peripheral blood at 16 weeks after transplantation. The lines represent average chimerism. $(\mathrm{H}, \mathrm{I})$ Transplantation assays showed the engraftment capacity of pre-HSC I (CD31 $\left.{ }^{+} \mathrm{CD} 41^{\text {low }} \mathrm{CD} 45^{-}\right)$and pre-HSC II $\left(\mathrm{CD} 31^{+} \mathrm{CD} 45^{+}\right)$of E11.5 WT AGM regions co-cultured with OP9-DL1 with or without CCTO07093 (CCT, $\left.25 \mu \mathrm{M}\right)$ for 4 days. **P<0.01. (J-M) Donor-derived multilineage output of myeloid $\left(\mathrm{Gr}-1^{+} / \mathrm{Mac1}^{+}, \mathrm{GM}\right), \mathrm{T}\left(\mathrm{CD}^{+}\right)$and $\mathrm{B}\left(\mathrm{B} 22 \mathrm{O}^{+}\right)$cells derived from pre-HSC I $(\mathrm{J})$ and pre-HSC II (K) of E11.5 WT and Wip1\% AGM region and of E11.5 WT AGM with or without the Wip1 inhibitor, CCT (L and M) at 16 weeks after transplantation. Ctr=dimethlysulfoxide control, $* P<0.05, * * P<0.01$. (N) Representative flow cytometric analysis of $\mathrm{CD} 31^{+} \mathrm{C}-\mathrm{Kit}^{\text {high }}\left(\mathrm{CD} 31^{+} \mathrm{C}-\mathrm{Kit}{ }^{\text {th }}\right)$ hematopoietic cluster cells in the E11.5 WT and Wip1\% AGM region. (0) Percentages and absolute numbers of $\mathrm{CD} 1^{+} \mathrm{C}-\mathrm{Kit}$ high cluster cells $(\mathrm{n}=9, * * * P<0.001)$. (P) Cell cycle status of endothelial cells (EC, CD31 $\left.{ }^{+} \mathrm{CD} 41^{-} \mathrm{CD} 45^{-}\right)$, pre-HSC I (I, CD31 $\left.{ }^{+} \mathrm{CD} 41^{\text {low }} \mathrm{CD} 45^{-}\right)$and pre-HSC II (II, CD31 ${ }^{+} \mathrm{CD} 45^{+}$) from WT and Wip1\% AGM regions was examined by Ki67 and 7-AAD staining ( $\mathrm{n}=3$, * $\left.P<0.05\right)$. WT: wildtype; KO: knockout; ee: embryo equivalent; GM: Gr-1+/Mac-1+ cells; T: CD3+ cells; B: B220+ cells. 
S5A, B). Perhaps Wip1 is involved in the endothelial to hematopoietic transition and hematopoietic cell expansion by regulating Runx1 and Gata2. To test which step in hematopoiesis Wip1 deletion was affected, we sorted EC (CD31 ${ }^{+} \mathrm{CD} 41^{-} \mathrm{CD} 45^{-}$Ter119-). The percentages of EC were indistinguishable between WT and Wip $1^{-1}$ AGM (Online Supplementary Figure S5C). Three-day co-cultures of OP9 and EC showed similar numbers of hematopoietic clusters, related to the earlier stage of endothelial to hematopoietic transition in Wip $1^{-1}$ AGM (Online Supplementary Figure $S 5 D, E$ ). However, after co-culture for 8 days, fewer $\mathrm{CD} 45^{+}$cells were found derived from Wip $^{-1}$ EC co-cultures (Online Supplementary Figure S5F), indicating that Wip1 mainly affects HPC maturation/expansion.

As previously reported, Wip1 influences the cell proliferation/cell cycle status of bone marrow HSC. ${ }^{11,12,15}$ Flow cytometric assays showed no differences in cell cycle status of total cells between E11.5 Wip $1^{-/}$and WT AGM. Conversely, significantly higher percentages of EC in G1 phase were observed after Wip1 deletion $(33.1 \pm 1.4 \%$ vs. $27.3 \pm 2.5 \%$ ), with no differences in proliferation. Furthermore, a lower percentage of pre-HSC I was in the G0 phase $(25.5 \pm 3.4 \%$ vs. $37.2 \pm 4.5 \%)$, however, the percentages of pre-HSC II in G1 phase were significantly higher in the Wip ${ }^{-1}$ AGM region $(40.0 \pm 3.1 \%$ vs. $31.26 \pm 2.6 \%$ ) (Figure $3 \mathrm{P}$ ). S/G2/M phases and proliferation status were not changed by Wip1 deficiency (Figure 3P, Online Supplementary Figure S6A-C). The apoptosis assay did not show any differences in the EC, pre-HSC I and II in Wip ${ }^{-1}$ AGM (Online Supplementary Figure S6D$6 F)$, suggesting influences of Wip1 on the cell cycle.

In summary, our study identifies a regulatory role for Wip1 in hematopoietic development during the mid-gestation stage. Transplantation assays after co-culture revealed that Wip1 regulates pre-HSC maturation in embryonic hematopoiesis, likely mediated by altered cell cycle status, laying a theoretical foundation for HSC regeneration in vitro.

Wenyan He, Xiaobo Wang, ${ }^{2}$ Yanli $\mathrm{Ni}^{2}$ Zongcheng $\mathrm{Li}^{2}$ Wei Liu, ${ }^{2}$ Zhilin Chang, ${ }^{2}$ Haowen Li, $^{1}$ Zhenyu Ju ${ }^{3}$ and Zhuan $\mathrm{Li}^{4}$

${ }^{1}$ China National Clinical Research Center for Neurological Diseases, Beijing Tiantan Hospital, Capital Medical University, Beijing; 'Laboratory of Oncology, Fifth Medical Center, General Hospital of PLA, Beijing; ${ }^{3}$ Key Laboratory of Regenerative Medicine of Ministry of Education, Guangzhou Regenerative Medicine and Health Guangdong Laboratory, Institute of Aging and Regenerative Medicine, Jinan University, Guangzhou and ${ }^{4}$ Department of Developmental Biology, School of Basic Medical Sciences, Southern Medical University, Guangzhou, China

Correspondence: ZHUAN LI - zhuanli2018@smu.edu.cn

ZHENYUJU - zhenyuju@163.com

doi:10.3324/haematol.2019.235481

Disclosures: no conflicts of interests to disclose.
Contributions: ZL, ZJ and WH designed research; WH performed research; $X W$ did some transplantation experiments; YN gave support on FACS; ZL supported bioinformatics analysis, WL, LC, HL prepared embryos; ZL and WH analyzed data; ZL, ZJ and WH wrote the paper.

Acknowledgments: we are grateful to Dr. L.A. Donehower from Baylor College of Medicine for the Wip1 knockout mice. We thank Dr. B. Liu and Dr. Y. Lan for useful discussions.

Funding: this work was supported by grants from the National Natural Science Foundation of China (81570095, 81870087, 91749203, 81525010), the National Key Research and Development Program (2019YFA0801802, SQ2019YFA0111100, 2016YFA0100602, 2017YFA0103302) and the Program for Guangdong Introducing Innovative and Entrepreneurial Teams (2017ZTO7S347) and the Innovative Team Program of Guangzhou Regenerative Medicine and Health Guangdong Laboratory (2018GZR110103002).

\section{References}

1. Muller AM, Medvinsky A, Strouboulis J, Grosveld F, Dzierzak E. Development of hematopoietic stem cell activity in the mouse embryo. Immunity. 1994;1(4):291-301.

2. Medvinsky A, Dzierzak E. Definitive hematopoiesis is autonomously initiated by the AGM region. Cell. 1996;86(6):897-906.

3. Li Z, Lan Y, He W, Chen D, et al. Mouse embryonic head as a site for hematopoietic stem cell development. Cell Stem Cell. 2012; 11(5):663-675

4. Li Z, Vink CS, Mariani SA, Dzierzak E. Subregional localization and characterization of Ly6aGFP-expressing hematopoietic cells in the mouse embryonic head. Dev Biol. 2016 ;416(1):34-41.

5. Ottersbach K, Dzierzak E. The murine placenta contains hematopoietic stem cells within the vascular labyrinth region. Dev Cell. 2005; 8(3):377-387.

6. Mikkola HK, Gekas C, Orkin SH, Dieterlen-Lievre F. Placenta as a site for hematopoietic stem cell development. Exp Hematol. 2005; 33(9):1048-1054.

7. Dzierzak E, Bigas A. Blood development: hematopoietic stem cell dependence and independence. Cell Stem Cell. 2018;22(5):639-651.

8. Chen MJ, Yokomizo T, Zeigler BM, Dzierzak E, Speck NA. Runx1 is required for the endothelial to haematopoietic cell transition but not thereafter. Nature. 2009;457(7231):887-891.

9. Bertrand JY, Chi NC, Santoso B, Teng S, Stainier DY, Traver D. Haematopoietic stem cells derive directly from aortic endothelium during development. Nature. 2010;464(7285):108-111.

10. Boisset JC, van Cappellen W, Andrieu-Soler C, Galjart N, Dzierzak E, Robin C. In vivo imaging of haematopoietic cells emerging from the mouse aortic endothelium. Nature. 2010;464(7285):116-120.

11. Chen Z, Yi W, Morita Y, et al. Wip1 deficiency impairs haematopoietic stem cell function via p53 and mTORC1 pathways. Nature Commun. 2015;6:6808

12. Schito ML, Demidov ON, Saito S, Ashwell JD, Appella E. Wip1 phosphatase-deficient mice exhibit defective $\mathrm{T}$ cell maturation due to sustained p53 activation. J Immunol. 2006;176(8):4818-4825.

13. Zhou F, Li X, Wang W, et al. Tracing haematopoietic stem cell formation at single-cell resolution. Nature. 2016;533(7604):487-492.

14. Yokomizo T, Dzierzak E. Three-dimensional cartography of hematopoietic clusters in the vasculature of whole mouse embryos. Development. 2010;137(21):3651-3661.

15. Uyanik B, Grigorash BB, Goloudina AR, Demidov ON. DNA damage-induced phosphatase Wip1 in regulation of hematopoiesis, immune system and inflammation. Cell Death Discov. 2017; 3:17018. 